\title{
Thermal Analysis of RF-MEMS Switches for Power Handling Front-end
}

\author{
F. Coccetti, B. Ducarouge, E. Scheid, D. Dubuc, K. Grenier, and R.Plana \\ LAAS-CNRS, 7. Avenue du Colonel Roche, 31077 Toulouse Cedex 4, France
}

\begin{abstract}
An experimental setup for the characterization of electromagnetic induced heat on MEMS devices undertaking high $\mathrm{RF}$ power regime $(>5 \mathrm{~W})$ is here proposed. The technique is based on infrared (IR) imaging of on-probe DUT, while it is in working conditions. The measured temperature distributions, for different working state of a RF-MEMS switch, are given. The results show that for a first considered capacitive switch, the most critical working state is the OFF-state (membrane actuated). In this case the hot-spots temperature reach $75.5^{\circ} \mathrm{C}$, for a input power of $6.3 \mathrm{~W}$ at $10 \mathrm{GHz}$. On the other hand, for the same incident power and frequency a maximum rise of only $5^{\circ} \mathrm{C}$ has been measured for the $\mathrm{ON}$ state (membrane in the rest position). Temperature mapping results for a second switch design are moreover presented. This steady-state map offers a real time global performance overview of the RF induced phenomena, and represents a very valuable real-time investigation tool for integrated MEMS and RFIC power handling front-end.
\end{abstract}

\section{INTRODUCTION}

Use of novel polymer based technology is emerging as very promising solution for integration on chip of wireless front-end. The excellent electric properties are however compromise by the low thermal conductivity, which represents a bottleneck in using such materials for high power handling (above $5 \mathrm{~W}$ ). The lack of heat sink could yield serious power handling limits, as thermomechanical deformation. This phenomenon becomes very serious in case of very compliant devices as MEMS varactors and switches, sensitive to the minimum displacements. Several efforts have been addressed to avoid power handling limitations by introducing separate actuation pads, or double control electrode, but very few have been dedicated to the experimental characterization [1]. So far RF induced heat due to joule effect, has been analyzed for RF-MEMS switches by simplified analytical and numerical models [2, 3]. A framework for the electromagnetic induced heat characterization by means of an experimental setup, which allows one to identify and quantify critical temperature rise (hot spots), can be a very useful tool for drawing power handling considerations and exploit them for design optimization. A characterization tool able to provide reliable experimental results directly on working devices could enable a very fast analysis of large systems as integrated front-end. The shrinking down (to micrometer scales) of reconfigurable antennae, phase shifters and switching networks, embedding MEMS functionalities, and bearing always higher RF signal power, could be investigated in real-time by means of the technique here reported.

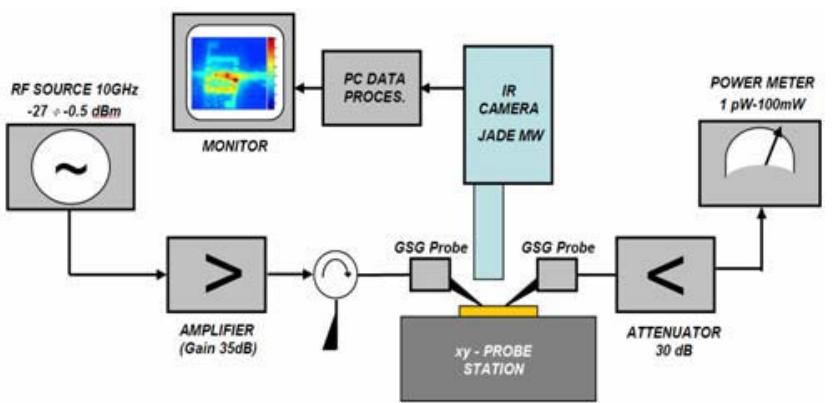

Fig. 1. Schematic of the setup used for the thermal imaging of RF-MEMS devices under working power handling conditions.

\section{EXPERIMENTAL SETUP}

The measurement set-up is composed of a RF source, delivering an RF signal which power can be vary from $27 \mathrm{dBm}$ up to $-0.5 \mathrm{dBm}$, followed by a solid state power, amplifier with $35 \mathrm{~dB}$ of gain in the frequency range 9.5$10.5 \mathrm{GHz}$. The maximum delivered power with the given source is of $39 \mathrm{dBm}$ on 50 Ohms. Due to cable loss however, the maximum incident power at the DUT is reduced to $6.3 \mathrm{~W}$. An isolator protects the amplifier in case of mismatching from spurious reflections along the signal path. A $30 \mathrm{~dB}$ attenuator and a power meter, with sensitivity $1 \mathrm{pW}-100 \mathrm{~mW}$, constitutes the receiving part of the measurement setup. The DUT, mounted between the isolator and the attenuator, can be any device with GSG probe access. The devices are realized on silicon wafer hold by a thermo-stabilized chuck, kept at the constant temperature of $23^{\circ} \mathrm{C}$. The thermal imaging is provided by the IR camera Jade MW [4], mounted on top of the probe station. The camera is characterized by a very cold detector, (93K) allowing very high thermal sensitivity. This is a critical parameter especially in case of very low emission material as gold (which emissivity ranges between 0.02 and 0.04 , depending whether it is polished or electroplated). The camera works in the spectral range of 2-5.2 $\mu \mathrm{m}$, and in the temperature range $20^{\circ} \mathrm{C}$ to $1300^{\circ} \mathrm{C}$. A special carrier makes it free of $\mathrm{x}-\mathrm{y}$ movements above the entire area of the wafer sample. A customized objective with focal length of $30 \mathrm{~mm}$ enables a resolution down to $20 \times 20-\mu m^{2}$ pixel (four times the estimated wavelength of the IR radiation). The IR camera is PC-assisted in order to carry out the numerical calibration and the results post processing. The entire measurement set up is shown in Fig.1. 


\begin{tabular}{|c|c|c|c|c|c|c|c|c|}
\hline \multirow{2}{*}{$\begin{array}{c}\text { Working } \\
\text { State }\end{array}$} & \multicolumn{4}{|c|}{ Temperature Rise $\boldsymbol{P}_{\text {in }}=3.6 W$} & \multicolumn{4}{c|}{ Temperature Rise $\boldsymbol{P}_{\text {in }}=6.3 W$} \\
\hline ON (Up) & Point 1 & Point 2 & Point 3 & Point 4 & Point 1 & Point 2 & Point 3 & Point 4 \\
\hline OFF (Down) & 2.5 & 2.5 & 2.4 & 2.4 & 5 & 3.6 & 5 & 5 \\
\hline
\end{tabular}

TABLE I

Temperature Rise at Steady State and at the Referenced Points (See Fig. 2)

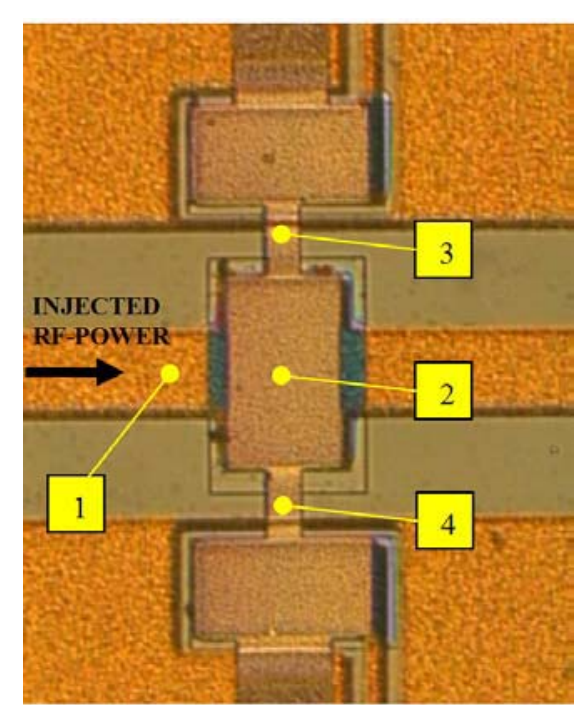

Fig. 2. Picture of the analyzed capacitive MEMS switch. The reference point for the temperature read out are shown along with the direction of incident RF signal.

The RF-MEMS device under test consists in a capacitive shunt switch,. The device is realized in gold on

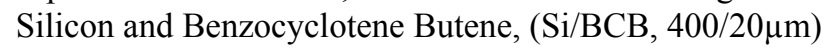
wafer, [5]. In Fig. 2 is given the picture for the analyzed device, indicating the direction of the incident RF power and the four points taken as reference and whereupon the temperature measurement have been carried out. Although the IR camera provides the map for the temperature over the entire region, the selection of few points is required for an optimal emissivity calibration.

\section{MEASUREMENTS RESULTS}

\section{A. Temperature Time Transient}

The measurements results for both working conditions (ON-OFF States), are given in Tab. I. The measurements have been performed at a frequency of $10 \mathrm{GHz}$, and by switching the incident RF power from 0.03 to $6.3 \mathrm{~W}$. For the device in ON-state (membrane up in rest position), the RF power signal transits through the MEMS discontinuity, with an overall maximum power loss given by

$$
L_{O N}=P_{i n}\left(1-\left|S_{11 O N}\right|^{2}-\left|S_{21 O N}\right|^{2}\right)=154 m W,
$$

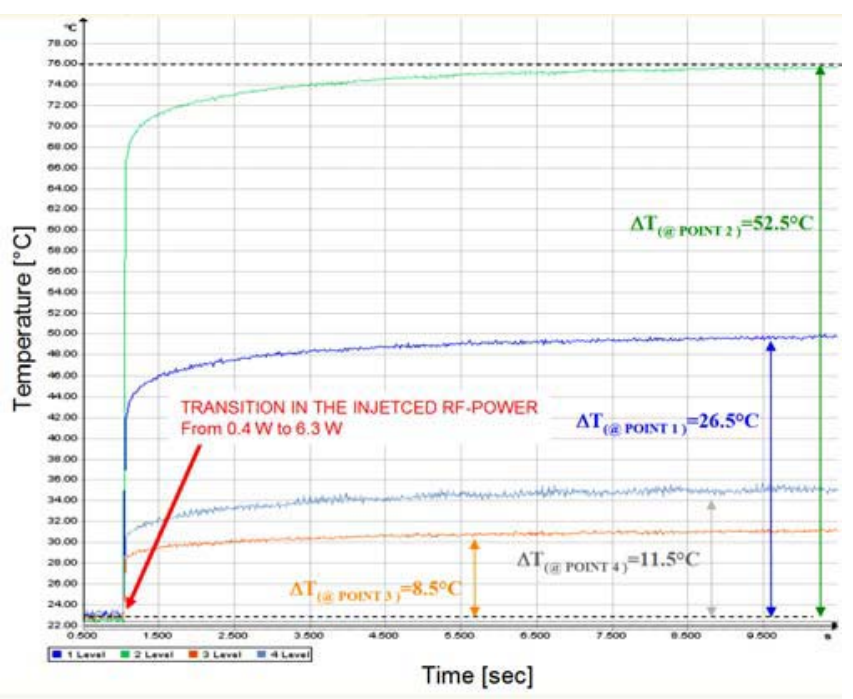

Fig. 3. Transient behaviour of the temperature rise for the four considered points. The RF incident power is turned from a minimum of $0.04 \mathrm{~W}$ to maximum of $6.3 \mathrm{~W}$ in correspondence of $1 \mathrm{sec}$. The device is at room temperature of $23^{\circ} \mathrm{C}$.

computed for a Pin $=6.3 \mathrm{~W}$, and the measured scattering parameters at $10 \mathrm{GHz},\left|S_{11 O N}\right|=-16.8 \mathrm{~dB}$, and $\left|S_{21 O N}\right|=-$ $0.2 d B$. The heat induced by Joule effect yields a temperature rise which reaches only $5^{\circ} \mathrm{C}$, quite uniformly all over the observed domain (only $1.4^{\circ} \mathrm{C}$ of difference between the considered points). The reference point temperatures are provided for two values of incident power $(3.6 \mathrm{~W}$ and $6.3 \mathrm{~W})$.

In case of working condition in OFF-state, (membrane actuated down), the loss due to the MEMS discontinuity and computed similarly to (1), are $L_{O F F}=532 \mathrm{~mW}$, (for the same incident power level Pin $=6.3 \mathrm{~W}$ and for measured scattering parameters at $10 \mathrm{GHz}$, equal to $\left|S_{\text {IIOFF }}\right|=-0.4$ $d B$, and $\left.\left|S_{21 O N}\right|=-22 d B\right)$. From the measurement results it is evident (see Tab I and Fig. 3) that the maximum temperature rise takes place in correspondence of the point 2, where the increment of temperature is of $52.5^{\circ} \mathrm{C}$. This can be justified by considering that the current distribution, responsible of the induced heat, has a maximum in correspondence of the membrane middle point (Point 2 see Fig. 2). It is there indeed that the capacitive virtual short takes place. The correspondent transient behaviour, showing the temperature evolution of the four considered points in time, once the input power is switched from 0.04 to $6.3 \mathrm{~W}$ is given in Fig. 3 .

Worth noting is that Point 3 and Point 4, corresponding to two symmetric positions on the gold membrane, present measured temperatures which differ for $4^{\circ} \mathrm{C}$ and $3^{\circ} \mathrm{C}$ each other, for $3.6 \mathrm{~W}$ and $6.3 \mathrm{~W}$. 


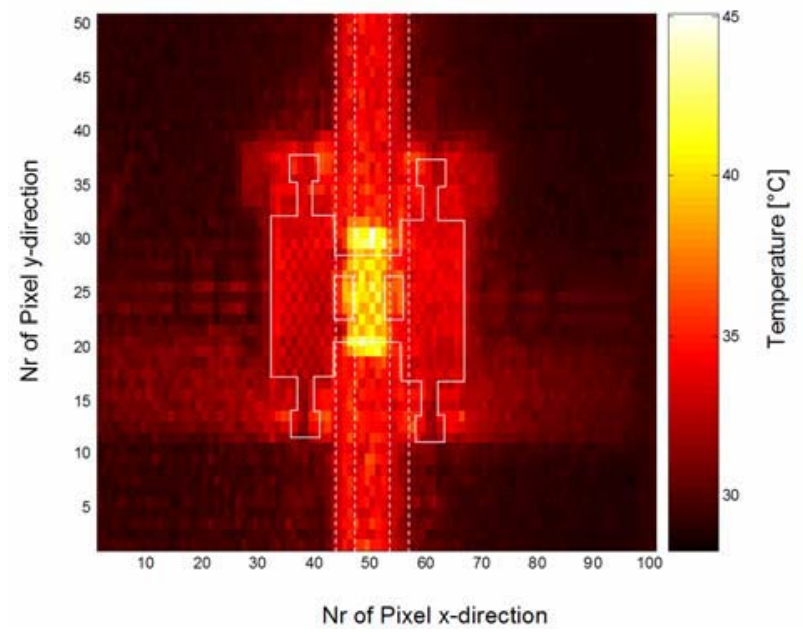

Fig. 4. ON-state (Membrane up) temperature map for the thermal steady-state in case $\mathrm{P}_{\text {in }}=6 \mathrm{~W}$ for the. The shape of the CPW line and of the membrane are denoted by the dashed and continuous line respectively. The maximum temperature is $45.2^{\circ} \mathrm{C}$.

respectively. This is likely due to the fact that in reality the structure presents slight asymmetries due to fabrication or actuation conditions, which yield the clearly observed temperature unbalance.

From this experimental observation can be drawn the conclusion that for the tested MEMS device the OFFstate (actuated membrane) results the most critical in terms of power handling. This is the opposite conclusion with respect to other research groups working on similar structure but different technology (similar devices but realized on $\mathrm{Si} / \mathrm{SiO}_{2}$ ) [2]. A possible explanation of this divergence is attributed to the critical role of material thermal properties. In the device here considered, the use of $20 \mu \mathrm{m}$ of BCB buffer layer between the silicon substrate and the metal structure yields a strong thermal isolation (the thermal conductivity of $\mathrm{BCB}$ is $0.29 \mathrm{~W} / \mathrm{m}$ $\mathrm{K}$, while the Silicon's is $148 \mathrm{~W} / \mathrm{m} \mathrm{K}$ ). In case of higher power loss in the MEMS region (as for switch in OFFState or membrane down), this impedes heat drain into the bulk substrate and causes temperature rise. MEMS directly built on Silicon, on the other hand suffer less of this limit. This suggests that improvement of the thermal drain through the $\mathrm{BCB}$ is necessary if we want to keep the device cool.

\section{B. Temperature map at steady state}

Once the DUT has reached the thermal steady state temperature distribution has been measured all over the region of interest (MEMS region). In figure Fig. 4 and Fig. 5, the results the two working states of a second MEMS switch design (but with same technology as the previous one), are shown. The most critical working state is the OFF-state with a maximum temperature of $84.5^{\circ} \mathrm{C}$ reached on the inner conductor in proximity of the membrane (which is in down in actuated position), on the side where the power is injected (top side in the figure). This confirms the previous measurements results.

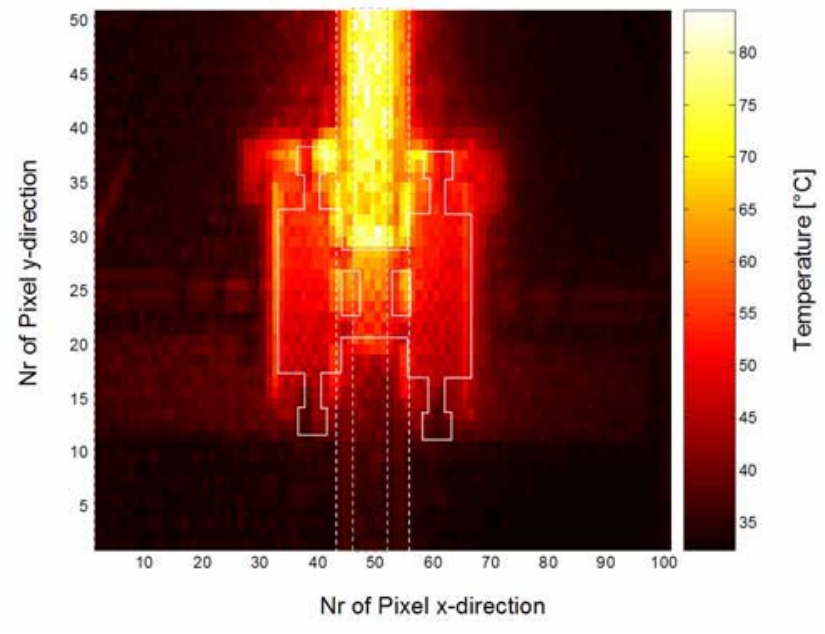

Fig. 5. OFF-state (membrane down) temperature map for the thermal steady-state in case of $P_{i n}=6 \mathrm{~W}$. The shape of the CPW line and of the membrane are denoted by the dashed and continuous line respectively. The maximum temperature is $84.5^{\circ} \mathrm{C}$.

Indeed the temperature distribution for the ON-state is more symmetric with respect of the signal flow and the maximum value does not go beyond $45.2^{\circ} \mathrm{C}$. From the temperature maps it become quite evident that very useful information as the location of the hot spots can be easily identified. The experimental results have been validated with a numerical modelling based on commercial tools (HFSS and e-physics from Ansoft Corp.) showing a very close agreement.

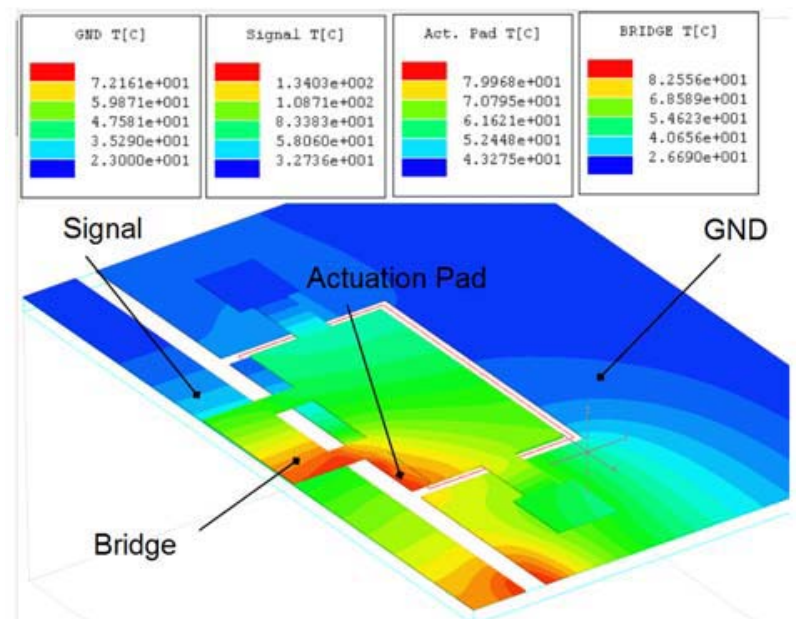

Fig. 6. OFF-state (membrane down) temperature map modelling results for input power $\mathrm{P}_{\mathrm{in}}=6 \mathrm{~W}$. The scale for the different metal parts are kept separate for the sake of clearness. The maximum temperature in the MEMS region is $82.6^{\circ} \mathrm{C}$.

This are based on the FEM method and gives the possibility to cope the electromagnetic problem into the thermal one, by reusing the power loss density due to conductor and dielectric derived from the full-wave electromagnetic solution [6]. For the ON and OFF-state working states the simulated temperature map a very similar qualitative distribution and maximum temperature of $56.3^{\circ} \mathrm{C}$ and $82.5^{\circ} \mathrm{C}$. Considering the difficulty in 
modelling the RF induced thermal problem for these device the results can be considered promising. The results for the only OFF-state are shown in Fig. 6 (the rise of temperature on the signal line at the input section is likely due to excitation fields).

\section{CONCLUSIONS}

In this paper an experimental setup which allows one to carry out thermal investigation of RF induced heat phenomena has been presented. The method enables the real-time investigation of RF-MEMS (so as RFICs) while under severe power regime working conditions.

The results for a capacitive MEMS switch have shown that for $6.3 \mathrm{~W}$ of $\mathrm{RF}$ incident signal at $10 \mathrm{GHz}$, the temperature rise reaches the $75.5^{\circ} \mathrm{C}$ in case of switch in $\mathrm{OFF}$ state, while remains below $28^{\circ} \mathrm{C}$ in the $\mathrm{ON}$-State for the same input signal. In both cases no electromagnetic performances degradation have been observed. However because of the linear proportionality between incident RF power and temperature [2], thermo-mechanical failures may be expected for the analysed device working in the OFF-State, and for input power above $10 \mathrm{~W}$. In this case, the hot spots temperature is expected to reach temperatures above $120^{\circ} \mathrm{C}$. Power handling design optimization could strongly benefit with the experimental insight provided by the presented technique. Especially in case of integrated Microsystems and front end, based on polymer technology (as BCB, Liquid crystal polymers or polyamide), real-time thermal imaging give immediate feedback on possible RF induced unwanted phenomena and represents immediate investigation tool for rapid failures inspection of planar MEMS so as RFIC devices.

\section{REFERENCES}

[1] M. Reano, K. Yang, J. F. Whitaker, and L. P. B. Katehi, "Simultaneous measurements of electric and thermal fields utilizing an electro-optic semiconductor probe," IEEE Trans. Microwave Theory Tech., vol. 49, pp. 2523-2531, Dec. 2001

[2] J. B. Rizk, E. Chaiban and G. M. Rebeiz, "Steady State Thermal Analysis and High-Power Reliability Considerations of RF MEMS Capacitive Switches," IEEE, MTT-S, TU3D-6, pp. 239-242, June 2002.

[3] W. Thiel, K. Tornquist, R. Reano and L.P.B. Katehi, "A Study of Thermal Effects in RF-MEM-Switches using a Time Domain Approach," IEEE, MTT-S, TU3D-5, pp. 235-238, June 2002.

[4] CEDIP Infrared Systems. www.cedip-infrared.com

[5] B. Ducarouge, D. Dubuc, S. Mellé, K. Grenier, L. Bary, P. Pons, and R. Plana, " Efficient design methodology of polymer based RF MEMS Switches," 5th IEEE Topical Meeting on Silicon Monolithic Integrated Circuits in RF systems (SiRF), Atlanta (USA), 8-10 Septembre 2004, pp.298-301

[6] Ansoft Corporation, www.ansoft.com 